\title{
Análise da morbimortalidade dos casos da COVID-19 nos estados brasileiros
}

\author{
Analisys of the morbimortality of COVID-19 cases in brazilian states \\ Análisis de la morbilidad y mortalidad de los casos de COVID-19 en estados brasileños \\ Sabrina Alves Praxedes ${ }^{1 *}$, Alexandra Ísis Soares de Lima Dantas ${ }^{1}$, Cyntia Mirelle Costa Lima ${ }^{1}$, \\ Luana Christie de Castro Medeiros ${ }^{1}$, Lethicia Beatriz Lima de Mesquita ${ }^{1}$, José Batista da Mota Neto ${ }^{1}$, \\ Layla Kathlien Ramos de Carvalho ${ }^{1}$, Hugo Moura Viana ${ }^{1}$, Ana Paula Nunes de Lima Fernandes².
}

\section{RESUMO}

Objetivo: Descrever e comparar variáveis de morbimortalidade dos casos de COVID-19 nos estados brasileiros. Métodos: Trata-se de um estudo ecológico descritivo e quantitativo. As informações coletadas foram obtidas no dia 14 de julho e agosto de 2020, por meio dos boletins epidemiológicos das Secretarias de Saúde dos 26 estados brasileiros e do Distrito Federal. Além disso, os elementos populacionais foram coletados do Instituto Brasileiro de Geografia e Estatística (IBGE). Os dados obtidos foram analisados por estatística descritiva e dispostos em gráficos, com auxílio do software Microsoft Excel 2016. Resultados: Foi evidenciada ampla divergência de letalidade, incidência e taxa de recuperação entre os estados brasileiros conforme o recorte temporal, com variação conforme aspectos socioeconômicos e sanitários, densidade demográfica, renda, integração às políticas de prevenção, bem como acesso à saúde pública. Conclusão: Desse modo, houve a explanação do conteúdo epidemiológico acerca da doença causada pelo novo coronavírus, permitindo uma reflexão comparativa entre os estados, a qual, dentro dos limites gerados pela subnotificação, foi capaz de caracterizar variáveis de morbidade e mortalidade, expressando valores que influenciam nas estratégias de intervenção da saúde pública relativas ao contexto da pandemia mundial.

Palavras-chave: Mortalidade, Morbidade, Epidemiologia, Coronavírus, COVID-19.

\begin{abstract}
Objective: To describe and compare morbidity and mortality variables of COVID-19 cases in Brazilian states. Methods: This is a descriptive and quantitative ecological study. The information collected was obtained on July 14 and August 2020, through epidemiological bulletins from the Health Departments of the 26 Brazilian states and the Federal District. In addition, population elements were collected from the Brazilian Institute of Geography and Statistics (IBGE). The data obtained were analyzed by descriptive statistics and arranged in graphs, with the aid of the Microsoft Excel 2016 software. Results: A wide divergence in lethality, incidence and recovery rate was evidenced among Brazilian states according to the time frame, with variation according to socioeconomic and sanitary aspects, demographic density, income, integration to prevention policies, as well as access to public health. Conclusion: Thus, there was an explanation of the epidemiological content about the disease caused by the new coronavirus, allowing a comparative reflection between the states, despite limits of underreporting, this article was capable of characterize morbidity and mortality variables, expressing values that influence public health intervention strategies related to the current context of the global pandemic.
\end{abstract}

Key words: Mortality, Morbility, Epidemiology, Coronavirus, COVID-19.

RESUMEN

Objetivo: Describir y comparar variables de morbimortalidad en casos de COVID-19 en estados brasileños. Métodos: Se trata de un estudio ecológico descriptivo y cuantitativo. Las informaciones colectadas fueran obtenidas en el día 14 de julio y agosto de 2020, a través de boletines epidemiológicos de los Departamentos

\footnotetext{
${ }^{1}$ Faculdade Nova Esperança de Mossoró (FACENE), Mossoró - RN.

*E-mail: sabrinaalpraxedes@gmail.com

2 Universidade Federal do Rio Grande do Norte (UFRN), Natal - RN.
} 
de Salud de los 26 estados brasileños y del Distrito Federal. Además, los elementos poblacionales fueran colectados del Instituto Brasileño de Geografía y Estatística (IBGE). Los datos obtenidos fueron analizados por medio de estadística descriptiva y representados en gráficos, con la ayuda del software Microsoft Excel 2016. Resultados: Fue evidenciada una amplia divergencia en la letalidad, incidencia y tasa de recuperación entre los estados brasileños según el marco temporal, con variación según los aspectos socioeconómicos y sanitarios, densidad demográfica, ingresos, integración con políticas de prevención, así como acceso a la salud pública. Conclusión: Por lo tanto, se explicó el contenido epidemiológico sobre la enfermedad provocada por el nuevo coronavirus, permitiendo una reflexión comparativa entre los estados, que dentro de los límites generados por el subregistro, fue capaz de caracterizar variables de morbilidad y mortalidad, expresando valores que inciden en las estrategias de intervención en salud pública relacionadas con el contexto de la pandemia global.

Palabras clave: Mortalidad, Morbilidad, Epidemiología, Coronavirus, COVID-19.

\section{INTRODUÇÃO}

A atual pandemia causada pela COVID-19 (do inglês, Coronavírus Disease 2019) está associada a uma Síndrome Respiratória Aguda causada pelo Novo Coronavírus (SARS-CoV-2), que se trata de uma doença principiada em Wuhan, na província de Hubei na China, que adoeceu milhões de pessoas em todo o mundo, a datar do final de novembro de 2019, quando a origem ainda era desconhecida (BRASIL, 2020).

A partir do dia 11 de março de 2020, a Organização Mundial de Saúde (OMS) categorizou uma pandemia pelo novo coronavírus. Hodiernamente, é sabido que o Sars-Cov-2 é um microrganismo altamente contagioso transmitido por meio do contato com gotículas e aerossóis respiratórios provenientes da secreção nasal ou oral de pessoas infectadas que, ao tossir, espirrar ou falar, contaminam o ambiente com as partículas virais. Ademais, quando o indivíduo entra em contato com superfícies contaminadas e toca suas mucosas como nariz, olhos e boca, também é possível desenvolver a infecção (NETTO RGF, et al., 2020).

O quadro clínico é típico de uma Síndrome Gripal com sintomas variando de febre $\left(>=37,8^{\circ} \mathrm{C}\right)$, tosse, dispneia, mialgia, fadiga, além de sintomas respiratórios superiores e gastrointestinais, como a diarreia, até uma apresentação grave, como choque séptico e falência respiratória que necessitam de atenção hospitalar para intubação e ventilação. A Síndrome Respiratória Aguda provocada pelo vírus varia entre casos leves, que correspondem a $80 \%$, e casos muito graves acompanhados de insuficiência respiratória, correspondendo entre $5 \%$ e $10 \%$. A doença possui período de incubação estimado de 5 a 6 dias, entretanto pode variar de 0 a 14 dias (BRASIL, 2020; CHAN JF, et al., 2020).

A dinâmica epidemiológica do número de casos, agravos e mortes provocadas pela doença do Sars-Cov2 na população desde o início dos primeiros casos na China, antes de se tornar pandemia, se faz extremamente necessária para o planejamento de políticas de saúde, de assistência e de controle da doença. Até a segunda quinzena de julho de 2020, havia cerca de quase 15 milhões de casos confirmados de COVID19 no mundo e mais de 600 mil mortes. Dos casos confirmados, mais da metade está situada no continente americano, sendo os Estados Unidos o país com maior número de casos e mortes pela doença, seguido do Brasil em ambos os dados (PLATERO K e GOMES F, 2020; WHO, 2020).

O primeiro caso confirmado no Brasil foi também o primeiro caso registrado da América Latina, sendo um caso importado e originado na Itália, tendo desembarcado na cidade de São Paulo - SP no dia 21 de fevereiro de 2020. Um mês após a confirmação do primeiro caso em território brasileiro, todos os demais estados também apresentaram notificações da COVID-19, já registrando óbitos em 7 desses estados. O vírus possui um coeficiente de letalidade no Brasil de, aproximadamente, 3,5\%, de acordo com dados extraídos da Plataforma Johns Hopkins no dia 27 de julho de 2020. Os casos de óbitos, em sua maioria, ocorreram em pacientes com condições de risco preexistentes como diabetes, hipertensão e doença respiratória crônica (NETTO RGF, et al., 2020; WANG C, et al., 2020).

A mortalidade pela Covid-19 varia de acordo com cada região brasileira, obedecendo variáveis como a densidade demográfica, o acesso à saúde pública e a condição socioeconômica. Além disso, a presença de comorbidades em pessoas infectadas, segundo estudos recentes, contribui significativamente para elevação 
da taxa de letalidade pela doença em questão. Segundo estudo realizado em Wuhan, cerca de $72 \%$ dos pacientes que foram internados em UTI devido ao novo coronavírus apresentavam comorbidades quando comparados aos que não precisavam de cuidados intensivos (DIETZ W e SANTOS-BURGOA C, 2020; NASCIMENTO IJB, et al., 2020; HUANG C, et al., 2020; SOUSA GJB, et al., 2020; XU XW, et al., 2020).

Sendo assim, os pacientes com comorbidades podem evoluir geralmente de forma rápida para a Síndrome do Desconforto Respiratório e outras emergências clínicas que necessitam de cuidados intensivos, eventos estes que podem resultar em morte. A partir dessas observações e de um estudo realizado com quase 60 mil pacientes de 11 diferentes países, as comorbidades mais associadas a mortalidade pela doença foram a hipertensão, diabetes, doença hepática e tabagismo (DIETZ W e SANTOS-BURGOA C, 2020; HUANG C, et al., 2020; XU XW, et al., 2020).

Tendo em vista o atual cenário pandêmico e a situação alarmante da pandemia no Brasil, caracterizada, principalmente, pelo número crescente de óbitos e pela superlotação dos serviços de atendimento em saúde, este estudo tem como objetivo analisar a morbimortalidade dos casos da COVID-19 nos estados brasileiros, mensurando e correlacionando as variáveis de saúde e sociodemográficas.

\section{MÉTODOS}

Trata-se de um estudo ecológico descritivo e comparativo com abordagem quantitativa. A amostra do estudo reúne o número de casos notificados, de óbitos e de pacientes recuperados, obtidos a partir dos informes epidemiológicos das Secretarias de Saúde dos 26 estados brasileiros e do Distrito Federal, publicados no dia 14 de julho e agosto de 2020. As variáveis selecionadas foram: taxa de recuperação, incidência e letalidade. Para cálculo da incidência, foram utilizadas as populações conforme o Instituto Brasileiro de Geografia e Estatística (IBGE), atualizado em 2019. Os elementos numéricos foram computados, analisados e dispostos em gráficos pelo programa Microsoft Excel versão 2016.

A pesquisa foi realizada em 3 etapas: a primeira deu-se pela coleta de dados de todos os informes epidemiológicos, no dia 14 de julho de 2020, nos quais foi observada convergência entre os estados nas informações referentes às variáveis: casos notificados, óbitos e pacientes recuperados, proporcionando a quantificação da letalidade, da incidência e da taxa de recuperação de cada unidade federativa. Dessa forma, para cálculo da incidência, foi efetuada a razão entre o número de pessoas que desenvolveram a doença no período e as pessoas-tempo em risco, multiplicada por $10^{\text {n }}$ (100 mil). Já a taxa de recuperação foi calculada a partir da divisão entre o número de recuperados e o número de doentes por determinada doença, multiplicada por 100. Por fim, efetuou-se a letalidade por meio da razão entre o número de mortes de uma determinada doença em certo período e o número de doentes por determinada doença no mesmo período, também multiplicada por 100 (BONITA R, et al., 2005).

No dia 14 de agosto, foi realizada a segunda coleta, constituindo a segunda fase da pesquisa, permitindo análise da evolução e regressão das variáveis estabelecidas em um mês de todos os estados brasileiros. Na terceira etapa, ocorrida no dia 21 de agosto, os dados foram dispostos em gráficos e tabelas para visualização límpida e interpretações epidemiológicas pertinentes. Vale salientar também que os dados usados são de natureza pública e não divulgam qualquer informação pessoal dos indivíduos que contribuíram como fonte de informações, não violando, portanto, as diretrizes do artigo 153 do Código Penal - Decreto Lei 2848/40 e da Lei 12527/11 (Lei de acesso à informação).

\section{RESULTADOS}

No que diz respeito à incidência a cada 100 mil habitantes dos casos de COVID-19 nos estados brasileiros no dia 14 de julho de 2020, é possível observar que as menores taxas registradas se referem ao Rio Grande do Sul (348), Minas Gerais (371) e Paraná (392). Em relação às federações brasileiras com as maiores incidências, estão em evidência os estados de Roraima (3.909), seguido por Amapá (3.766) e Distrito Federal (2.442). No que se refere ao número de casos, os dados coletados a partir dos informes epidemiológicos mostram que o estado de São Paulo possui o maior número de infectados, respectivamente com 389.607 
contaminados, seguido do Rio de Janeiro, com 132.822 notificações e do Ceará, com 145.797 confirmações. As federações com os menores números de casos foram Mato Grosso do Sul (13.934), Tocantins (15.723) e Acre (16.479) (Gráfico 1).

Gráfico 1 - Incidência a cada 100 mil habitantes dos casos da COVID-19 das federações brasileiras e do Distrito Federal no dia 14 de julho de 2020 desde o início da pandemia.

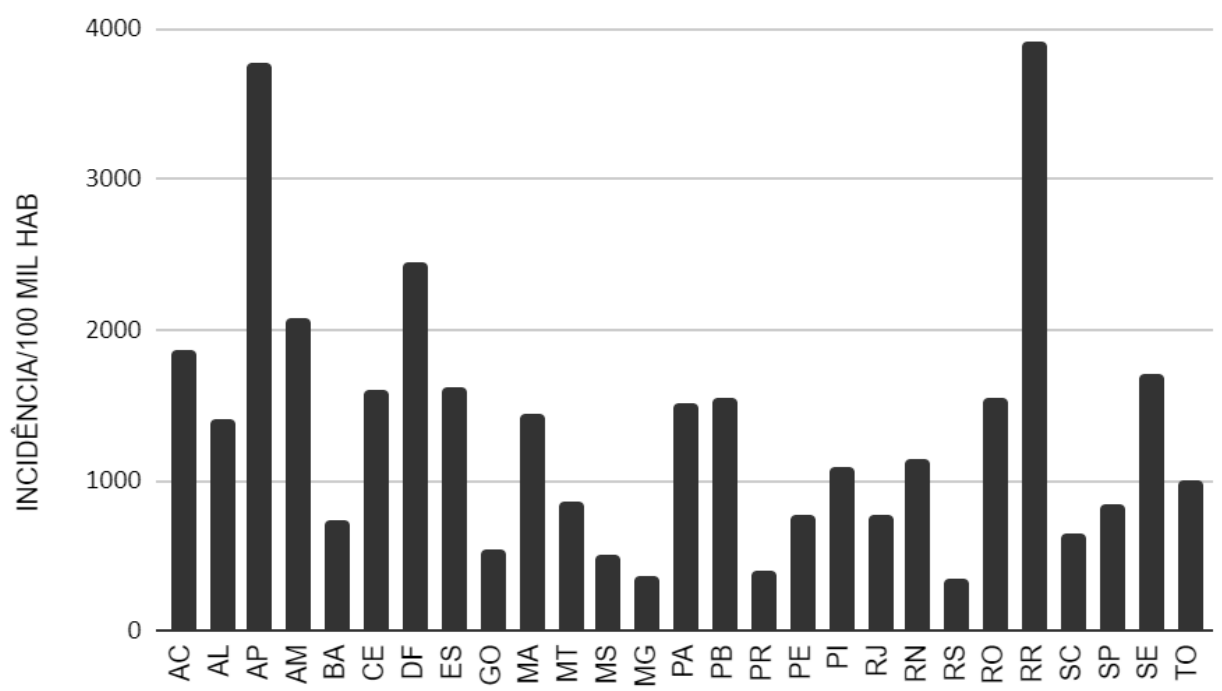

Fonte: Praxedes SA, et al., 2020; dados extraídos das Secretarias Estaduais de Saúde brasileiras.

No que tange a taxa de recuperação notificada nos informes das Secretarias Estaduais de Saúde no dia 14 de julho de 2020, o estado com maior taxa de recuperados é o Rio Grande do Sul (97,49\%), seguido pelo Mato Grosso (96,13\%), Piauí $(94,66 \%)$, Pará $(88,48 \%)$ e Rio de Janeiro $(84,59 \%)$. Em contrapartida, as federações com menores porcentagens de recuperação são o Rio Grande do Norte (8,08\%), Goiás $(30,07 \%)$, Paraná (34,06\%), Roraima (34,6\%) e Paraíba (36,87\%) (Gráfico 2).

Gráfico 2 - Taxa de recuperação (\%) dos pacientes com COVID-19 das federações brasileiras e do Distrito Federal no dia 14 de julho de 2020 desde o início da pandemia.

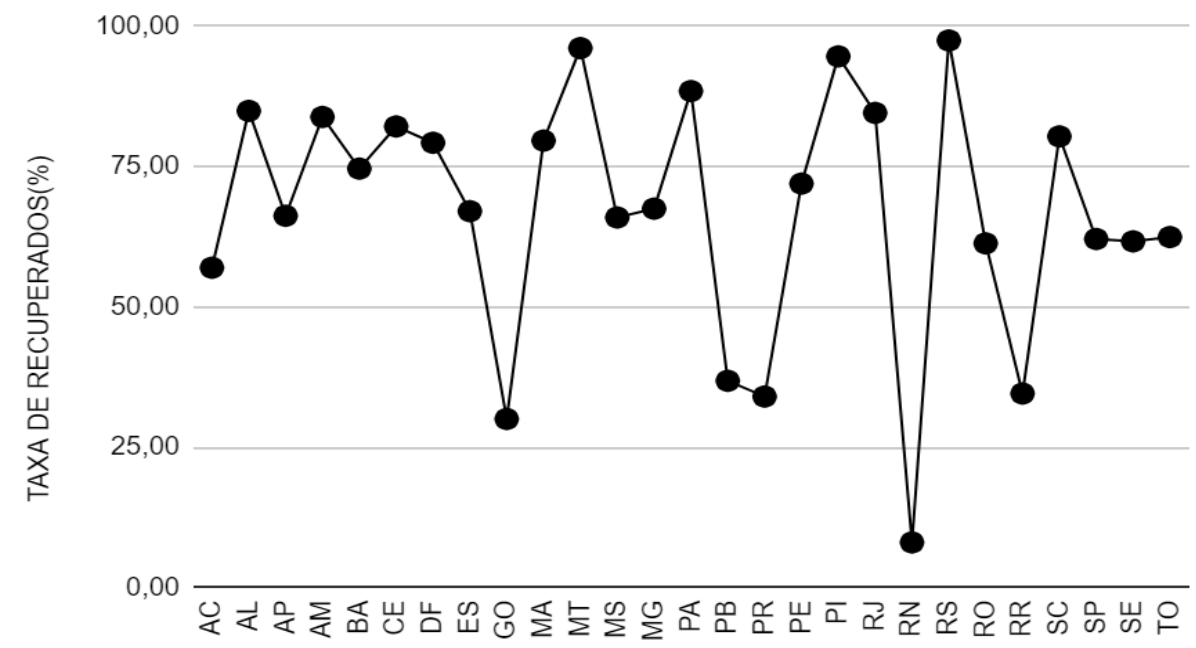

Fonte: Praxedes SA, et al., 2020; dados extraídos das Secretarias Estaduais de Saúde brasileiras. 
No que se refere a letalidade demonstrada graficamente acima, podemos visualizar que os estados que possuem maiores taxas em percentual são o Rio de Janeiro $(8,76 \%)$, seguido da Paraíba $(8,54 \%)$ e Pernambuco (7,77\%). O estado do Ceará ainda se apresenta em quarto colocado em letalidade, com 4,9\%, seguido por São Paulo com 4,74\%. Evidenciam-se, também, as menores letalidades, ocorridas nos estados de Santa Catarina (1,16\%) e Mato Grosso do Sul $(1,27 \%)$, bem como o Distrito Federal $(1,3 \%)$, seguindo o Amapá (1,52\%), Roraima (1,7\%) e Tocantins (1,7\%) (Gráfico 3).

Gráfico 3 - Letalidade (\%) das federações brasileiras e do Distrito Federal pela COVID19 no dia 14 de julho de 2020 desde o início da pandemia.

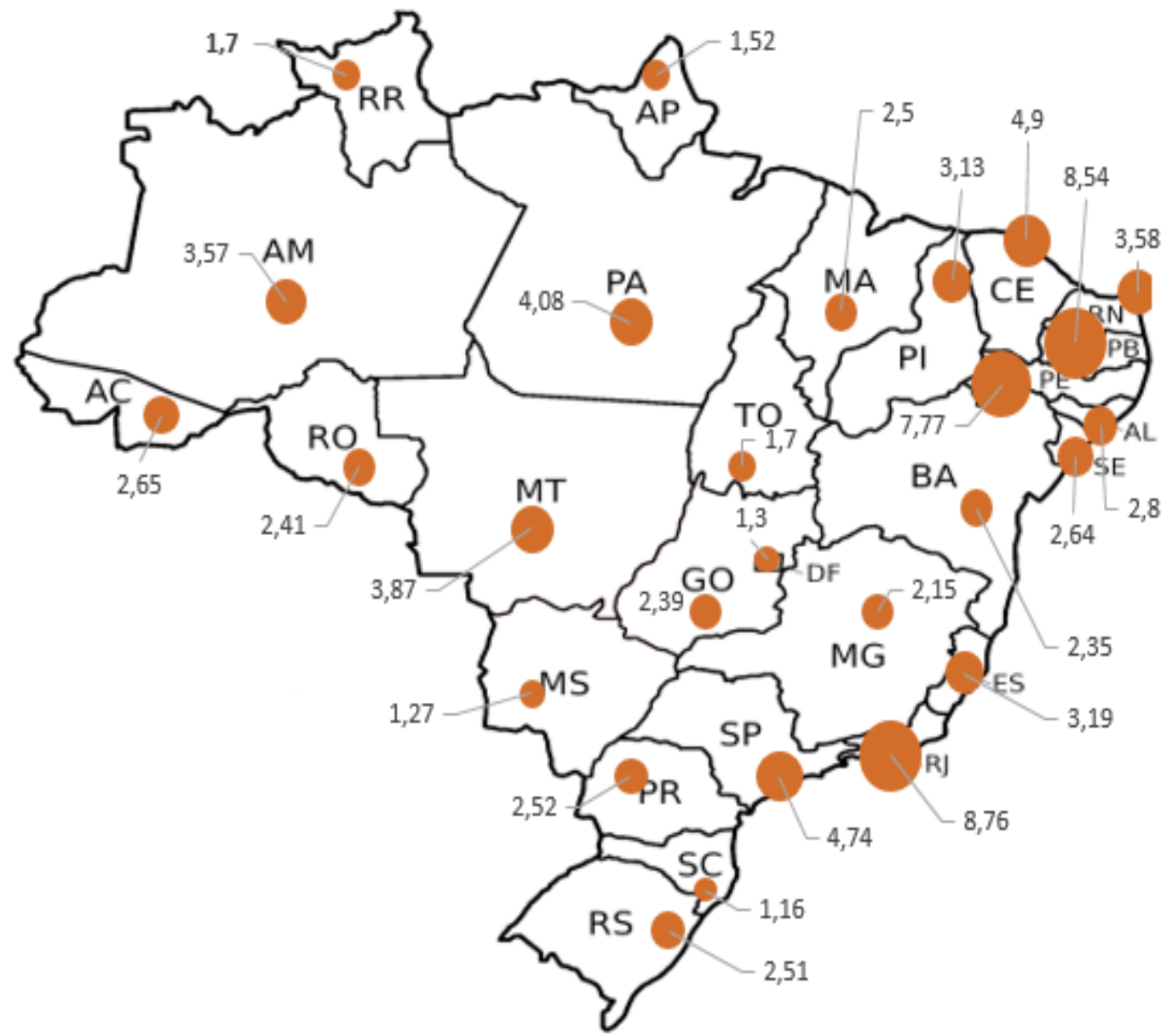

Fonte: Praxedes SA, et al., 2020; dados extraídos das Secretarias Estaduais de Saúde brasileiras.

Ademais, no que tange a incidência a cada cem mil habitantes dos casos de COVID-19 no dia 14 de agosto de 2020, é empírico que os estados que tiveram maiores valores foram Roraima $(6503,72)$, Amapá $(4640,25)$ e o Distrito Federal $(4416,39)$, os quais continuam na mesma colocação em relação ao gráfico do dia 14 de julho. Ainda entre os primeiros colocados no ranking de incidência, Sergipe $(2924,48)$, Amazonas $(2672,81)$ e Rondônia $(2660,78)$. Já os que tiveram os menores valores foram Minas Gerais $(795,64)$, Rio Grande do Sul $(836,25)$, Paraná $(880,85)$, Rio de Janeiro $(1099,86)$ e Pernambuco $(1155,26)$. No tangente aos casos confirmados, o estado com maior número total de infectados segue sendo São Paulo, no momento com 674.455 casos. Além disso, são também destaque nesse ranking Bahia (210.993 casos), Rio de Janeiro (189.891), Pará (177.010) e Minas Gerais (168.428). As federações com menor número de casos totais são Acre (22.435), Amapá (39.244), Tocantins (25.403), Mato Grosso do Sul (35.434) e Rondônia (47.288) (Gráfico 4). 
Gráfico 4 - Incidência a cada 100 mil habitantes dos casos da COVID-19 das federações brasileiras e do Distrito Federal no dia 14 de agosto de 2020 desde o início da pandemia.

8000

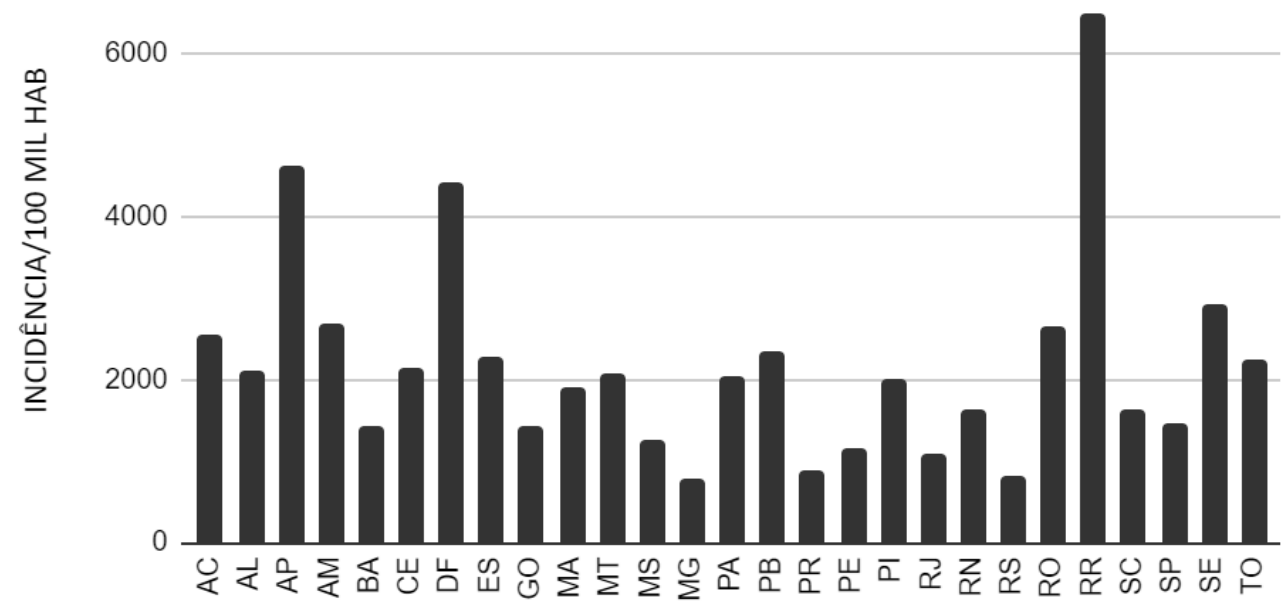

Fonte: Praxedes SA, et al., 2020; dados extraídos das Secretarias Estaduais de Saúde brasileiras.

Outrossim, a taxa de recuperação dos pacientes de COVID-19 no mês de agosto de 2020, em porcentagem, demonstrada no gráfico acima evidenciam que os Estados da Bahia (90,44\%), Pará (90,98\%), Maranhão (91,66\%), Alagoas $(92,55 \%)$ e Piauí $(96,57 \%)$ são os que tiveram maior taxa de recuperação proporcional a seus habitantes. Já os que possuem as menores taxas de recuperação são o Paraná (61,89\%), Tocantins (61,85\%), Rio Grande do Norte (59,49\%), Paraíba (53,17\%) e Roraima (23,84\%) (Gráfico 5).

Gráfico 5 - Taxa de recuperação (\%) dos pacientes com COVID-19 das federações brasileiras e do Distrito Federal no dia 14 de agosto de 2020 desde o início da pandemia.

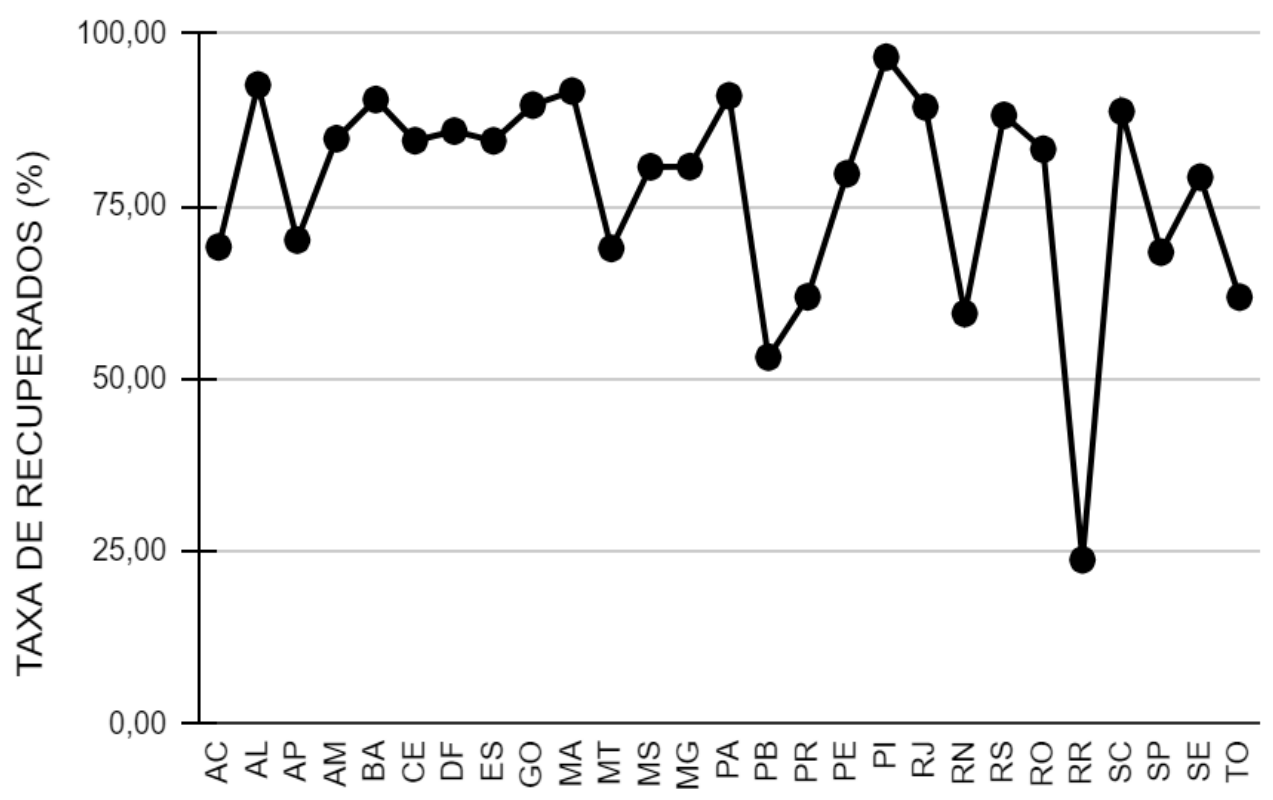

Fonte: Praxedes SA, et al., 2020; dados extraídos das Secretarias Estaduais de Saúde brasileiras. 
No tangente à evolução do número de mortos por habitantes dos estados para o dia 14 de agosto, explicitou-se que as maiores letalidades são do Rio de Janeiro (7,64\%), Pernambuco $(6,44 \%)$ e Distrito Federal $(6,1 \%)$, Ceará $(4,13 \%)$ e Rio Grande do Norte $(3,55 \%)$. No que se refere aos estados mais promissores, foi evidenciado que Tocantins $(1,41 \%)$, Roraima $(1,44 \%)$, Santa Catarina $(1,47 \%)$, Sergipe $(1,51 \%)$ e Amapá $(1,56 \%)$ apresentam menor número de mortes a cada 100 mil habitantes (Gráfico 6 ).

Gráfico 6 - Letalidade (\%) das federações brasileiras e do Distrito Federal pela COVID-19 no dia 14 de agosto de 2020 desde o início da pandemia.

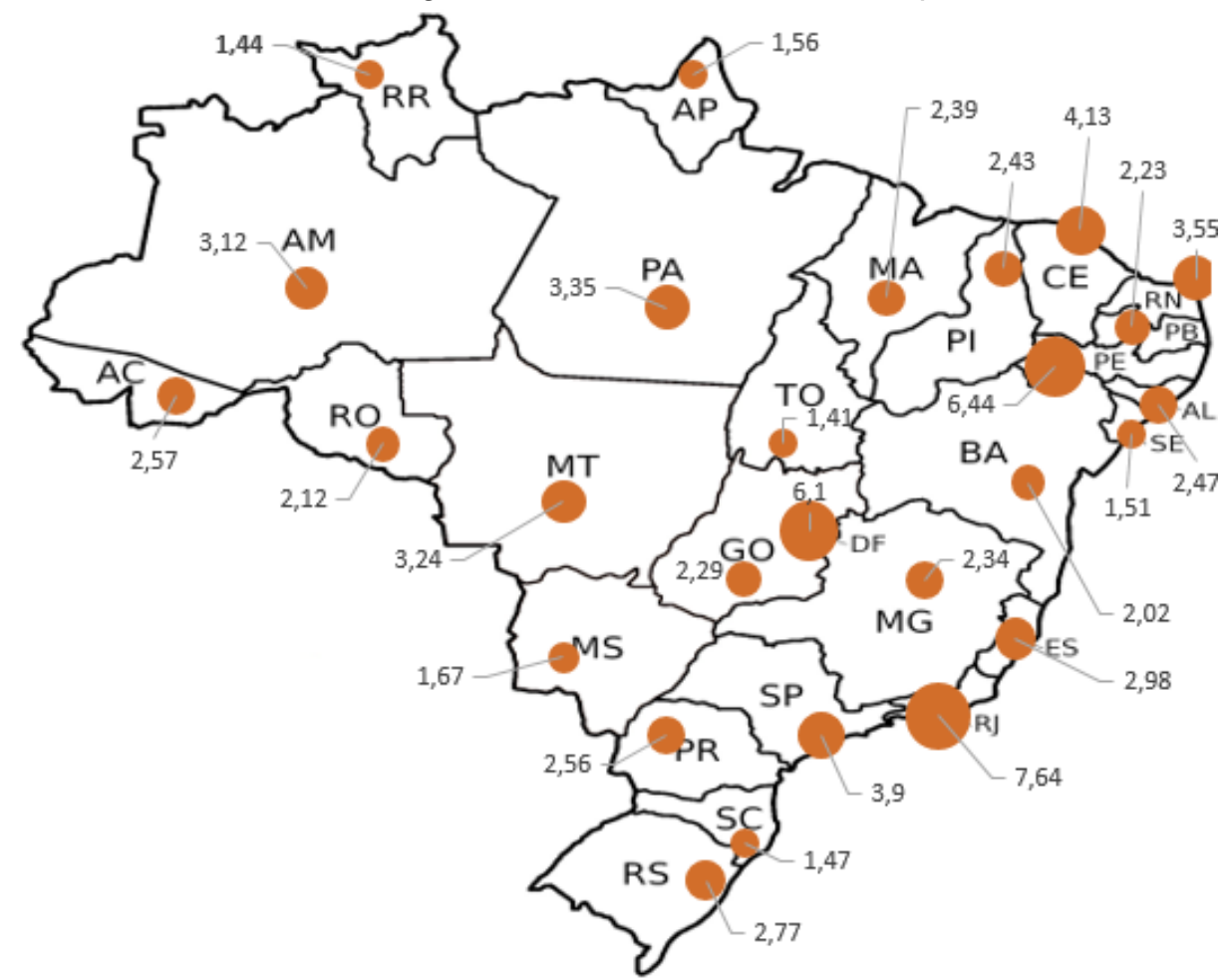

Fonte: Praxedes SA, et al., 2020; dados extraídos das Secretarias Estaduais de Saúde brasileiras.

\section{DISCUSSÃO}

Dentre os cinco estados com maior taxa de letalidade do Brasil, três são da região Nordeste, sendo eles: Paraíba, Pernambuco e Ceará. As Regiões Norte e Nordeste sofrem por uma cronificação da vulnerabilidade social, justificando o fato dessas unidades federativas possuírem as maiores taxas de letalidade pela COVID19 do país. Nesse sentido, entre a décima terceira e décima sétima semana epidemiológica, o Brasil possuía 32.757 leitos de UTI para adultos, dos quais 45,4\% (14.873) pertencem ao Sistema Único de Saúde (SUS). Desse total, o Piauí e a Paraíba - que ocupam as primeiras posições no ranking de letalidade - possuem apenas $0,69 \%$ (227) e 1,38\% (454) dos leitos da disponibilidade nacional, respectivamente, ou seja, somente 6,9 leitos/100 mil habitantes e 11,2 leitos/100 mil habitantes (SOUZA CDF, et al., 2020).

$\mathrm{Na}$ Região Norte, o Amazonas dispõe apenas de 271 leitos de UTI, resultando em um total de 6,5 leitos para 100 mil habitantes. Contudo, sabe-se que são diversos os fatores que tornam a população de baixa renda mais sujeita à infecção pelo novo coronavírus, além da falta de leitos e auxílio terapêutico, tais como: o uso de transporte público, a maior quantidade de moradores por domicílio, a precariedade de saneamento básico, bem como a falta de isolamento social devido a extrema necessidade do trabalho para manutenção da renda (ANGELO JR, et al., 2020; SOUZA CDF, et al., 2020).

Em adição, é importante destacar que esses territórios concentram grupos de indivíduos que apresentam condições de saúde já comprometidas, a exemplo da alta prevalência de tuberculose, hipertensão, 
cardiopatias e diabetes, além das resultantes da violência, expressas por elevadas taxas de homicídios, que proporcionam maior incidência de casos graves. Outrossim, foi visto que o Rio Grande do Norte, no Gráfico 2, obteve o menor número de recuperações dentre todas as federações, agravando o cenário. Apesar disso, é importante ressaltar que pode ter ocorrido uma subnotificação nos dados apresentados, tendo como consequência a discrepante exposição de dados quando comparado aos outros estados (ANGELO JR, et al., 2020).

Como exposto nos Gráficos 3 e 6, o estado do Rio de Janeiro é o líder em letalidade por COVID-19, demonstrando a importância de discorrer acerca da pobreza urbana, a qual, veementemente, promove desigualdade e permite o avanço mais rápido do novo coronavírus nas localidades de maior instabilidade social, cultural e econômica. Isso pode ser explicado a partir da falta de políticas públicas que proporcionem suporte à proteção coletiva, ações educativas, sociais e proteção à saúde pública estadual (SOUZA CDF, et al., 2020).

Haja vista tais fatores, é importante salientar que o número de casos e óbitos pela COVID-19 também são determinados pelas características intrínsecas dos indivíduos infectados (a saber: idade, doenças prévias, hábitos de vida), quanto pela oferta recursos terapêuticos (equipes de saúde, ventiladores mecânicos medicamentos e leitos). Por esse motivo, a avaliação da letalidade deve correlacionar os diversos elementos com potencial regressivo, tendo em vista uma intervenção complexa e prática das equipes de saúde nas comunidades (SOUZA CDF, et al., 2020).

Na segunda etapa da pesquisa, realizada no dia 14 de agosto, os estados nordestinos que permanecem na posição de destaque na variável "letalidade" são Pernambuco em segundo lugar e Ceará em quarto, ambos abaixo estatisticamente do Rio de Janeiro. Contudo, explicita-se o decréscimo da quantidade de mortes em relação ao número de casos contabilizados nas unidades federativas citadas em um mês, caracterizando uma diminuição proporcional no número de mortes. Ademais, as medidas já implantadas em algumas cidades e estados brasileiros, como cancelamento de aulas em todos os níveis, distanciamento social e quarentena voluntária, têm estimulado uma redução no número de casos de infecção. No entanto, devido a questões econômicas anteriormente mencionadas, há no presente momento uma pressão popular para a suspensão de tais medidas (CANABARRO A, et al., 2020; PIRES LN, et al., 2020).

Conforme apresentado nos Gráficos 3 e 6, Santa Catarina (SC) apresenta baixa letalidade quando comparada às demais unidades federativas, tanto em julho como em agosto. A federação em questão está dentre os estados com o maior índice de desenvolvimento humano (IDH) do país, visto que esse índice considera a longevidade, a educação e o desenvolvimento econômico para cálculo. Dessa maneira, é possível afirmar que tais fatores, de acordo com os resultados exibidos no artigo, estão intrinsecamente relacionados aos problemas de saúde pública relativos às medidas de combate ao novo coronavírus. Tal lógica também se aplica aos demais estados sulistas com números mais promissores em relação à patologia (IPEA, 2010).

Sendo assim, o Rio Grande do Sul e o Mato Grosso também estão inclusos como os estados de melhor classificação no ranking do IDH, ao passo que Rio Grande do Norte, Roraima e Goiás apresentam índices menores. Logo, infere-se que o baixo IDH reflete na dificuldade de adoção de estratégias de prevenção por parte da população. Sendo assim, fragilidades sanitárias e socioeconômicas, inerentes às características das unidades federativas, levam à violação da população urbana economicamente desfavorecida no contexto da pandemia mundial (IPEA, 2010; SOUZA CDF, et al., 2020).

Contudo, é válido destacar que as unidades federativas da Região Sul (Rio Grande do Sul, Santa Catarina e Paraná) possuem determinantes importantes de letalidade, a exemplo: os maiores índices de envelhecimento do país, maior incidência histórica de síndrome respiratória aguda grave e uma rede de saúde fragilizada, ainda que mais estruturada quando comparada às demais regiões brasileiras. Não obstante, a proximidade dessas localidades com Rio de Janeiro e São Paulo incita o deslocamento de pessoas entre os estados, caracterizando um fator complicador no combate à doença (SOUZA CDF, et al., 2020).

Em contrapartida, nas duas coletas de dados, as maiores incidências se dão em Roraima, Amapá e no Distrito Federal. Já as menores incidências se dão até o momento no Rio Grande do Sul, Minas Gerais e 
Paraná. Isso pode ser justificado, além das considerações já levantadas, devido a implementação das medidas de distanciamento social, como suspensão de eventos e de aulas, e quarentena de grupos de risco antes do $10^{\circ}$ caso confirmado nos estados do Rio Grande do Sul, Minas Gerais e Paraná, por exemplo (SILVA LLS, et al., 2020).

No que tange às limitações do estudo, é importante ressaltar que existe uma influência direta da subnotificação da doença e de óbitos, visto que estados com baixa quantidade de testagem tendem a possuir maiores taxas de letalidade, pois os dados não apresentam o número exato de enfermos. Além disso, há um elevado número de óbitos que não tiveram sua causa confirmada, principalmente entre idosos, os quais constantemente têm taxas de necropsias reduzidas, o que também compromete a qualidade dos registros (MIRANDA GMD, et al., 2016; SOUZA CDF, et al., 2020).

Dessa maneira, estudos recentes têm sugerido que o número de infectados no país pode ser de 10 a 15 vezes maior do que o número de casos notificados. Nesse sentido, não há conhecimento da verdadeira amplitude da pandemia, o que representa uma barreira contra a mitigação da problemática. Ademais, localidades com menor número de habitantes tendem a ter maiores incidências devido a desproporcionalidade do número de testagens entre os estados (SOUZA CDF, et al., 2020).

Os dados provenientes das estatísticas de mortalidade são afetados por diversas fontes de erros, porém, dentro de um cenário epidemiológico pandêmico, fornecem dados pertinentes sobre o estado de saúde das populações. O emprego dos dados depende, entre outros fatores, do apropriado preenchimento dos registros e da acurácia na determinação da causa básica do óbito. Os epidemiologistas utilizam com frequência as estatísticas de mortalidade para avaliar a carga de doenças nas populações e, ainda, para avaliar modificações na ocorrência de doenças no decorrer dos anos. Porém, em muitos países, as estatísticas básicas de mortalidade não estão disponíveis, em geral devido à escassez de recursos para estabelecer um sistema rotineiro de registro vital. Outrossim, o fornecimento de informações acuradas sobre a causa da morte é prioridade para os serviços de saúde (BONITA R, et al., 2005).

No intuito de aperfeiçoar os registros epidemiológicos acerca do novo coronavírus, é indicada a mensuração de dados de saúde e a realização de estudos estatísticos detalhados, a fim de explanar o conhecimento voltado às necessidades da população acerca da patologia quanto às diversas variáveis, como sexo, idade e comorbidades.

\section{CONCLUSÃO}

O presente estudo mostrou que as regiões brasileiras de maior vulnerabilidade social e econômica são potencialmente suscetíveis a maiores valores de letalidade e incidência, bem como a taxas de recuperação mais baixas, o que decorre da inacessibilidade às políticas públicas, desigualdades culturais, sociais, econômicas e estruturais. Ademais, é empírico que a letalidade tende a diminuir percentualmente na maioria dos estados no recorte temporal estipulado, além de ser inferior à taxa de curados. Apesar disso, o número de casos cresce incontrolavelmente, devido ao alto índice de transmissibilidade do vírus, uma vez que as ações de prevenção orientadas, a exemplo do isolamento social, não estão sendo cumpridas, o que promoverá a decadência do sistema de saúde. Portanto, o aumento do número de casos de COVID-19 no país é coerente com a mudança de perfil socioeconômico da população e com a acessibilidade às redes de atenção à saúde de cada região brasileira.

\section{REFERÊNCIAS}

1. ANGELO JR, et al. Boletim socioepidemiológico da Covid nas favelas: análise da frequência, incidência, mortalidade e letalidade por COVID-19 em favelas cariocas. Rio de Janeiro: Fiocruz, 2020; 1(2): 1-47.

2. BONITA R, et al. Basic Epidemiology. Lancet, 2005; 365(1099): 1005.

3. BRASIL. Ministério da Saúde. Secretaria de Atenção Primária à Saúde. Protocolo de Manejo Clínico do Coronavírus (COVID-19) na Atenção Primária à Saúde. Brasília, Mai. 2020; 9(1): 35-40. 
4. CANABARRO A, et al. Data-Driven Study of the COVID-19 Pandemic via Age-Structured Modelling and Prediction of the Health System Failure in Brazil amid Diverse Intervention Strategies. MedRxiv, 2020; 15(7): 1-13.

5. CHAN JFW, et al. A familial cluster of pneumonia associated with the 2019 novel coronavirus indicating person-toperson transmission: a study of a family cluster. The Lancet, 2020; 395(10223): 514-523.

6. DIETZ W, SANTOS-BURGOA C. Obesity and its implications for COVID-19 mortality. Obesity (Silver Spring), 2020; 28(6): 1005.

7. HUANG C, et al. Clinical features of patients infected with 2019 novel coronavirus in Wuhan, China. The lancet, 2020; 395 (10223): 497-506.

8. IBGE. INSTITUTO BRASILEIRO DE GEOGRAFIA E ESTATÍstICA. Cidades e estados. 2020. Disponível em: https://www.ibge.gov.br/cidades-e-estados. Acesso em: 27 Jul. 2020.

9. IPEA. INSTITUTO DE PESQUISA ECONÔMICA APLICADA. Brasil em desenvolvimento: Estado, planejamento e políticas $\quad$ públicas. $\quad$ Brasília: $2010 . \quad$ Disponível https://www.ipea.gov.br/portal/images/stories/Livro_BrasilDesenvEN_Vol01.pdf. Acesso em: 28 Jul. 2020.

10. MIRANDA GMD, et al. Population aging in Brazil: current and future social challenges and consequences. Revista Brasileira de Geriatria e Gerontologia, 2016; 19(3): 507-519.

11. NASCIMENTO IJB, et al. Novel coronavirus infection (COVID-19) in humans: a scoping review and meta-analysis. Journal of clinical medicine, 2020; 9(4): 941.

12. NETTO RGF, CORREA JWN. Epidemiologia do surto de doença por coronavírus (COVID-19). DESAFIOS - Revista Interdisciplinar da Universidade Federal do Tocantins, 2020; 7(3): 18-25.

13. PIRES LN, et al. COVID-19 e desigualdade: a distribuição dos fatores de risco no Brasil. Research Gate, 2020; 21(10): $1-4$

14. PLATERO K, GOMES F. Números estatísticos e realidades: Uma proposta de reflexão sobre a pandemia de Covid19 no Brasil. DILEMAS: Revista de Estudos de Conflito e Controle Social-Reflexões na Pandemia, 2020; 1-11.

15. SILVA LLS, et al. Medidas de distanciamento social para o enfrentamento da COVID-19 no Brasil: caracterização e análise epidemiológica por estado. Cadernos de Saúde Pública, 2020; 36(9): 1-15.

16. SOUSA CDF, et al. Evolução espaçotemporal da letalidade por COVID-19 no Brasil. Jornal Brasileiro de Pneumologia, 2020; 46(4): 1-3.

17. SOUSA GJB, et al. Estimação e predição dos casos de COVID-19 nas metrópoles brasileiras. Revista LatinoAmericana de Enfermagem, 2020; 28(3345): 2-8.

18. WANG C, et al. A novel coronavirus outbreak of global health concern. Lancet, 2020; 395(10223): 470-473.

19. WHO. WORLD HEALTH ORGANIZATION. WHO coronavirus disease (Covid-19) Dashboard. 2020. Disponível em: https://apps.who.int/iris/bitstream/handle/10665/336034/nCoV-weekly-sitrep11Oct20-eng.pdf. Acesso em: 28 Jul. 2020.

20. XU XW, et al. Clinical findings in a group of patients infected with the 2019 novel coronavirus (SARS-Cov-2) outside of Wuhan, China: retrospective case series. bmj, 2020; 368(606): 1-7. 